\title{
Nível de conhecimento, atitudes e práticas dos manipuladores de alimentos em serviços de alimentação
}

\section{Knowledge, attitudes and practices of food handlers in food services}

Hiara Zanoni Pagotto ${ }^{\top}$

Ludmilla Gonçalves Espíndula

Alyne Gomes da Vitória ${ }^{2}$

Maria Clara de Moraes Motta Machado2

Jackline Freitas Brilhante de São José ${ }^{2}$

' Universidade Federal do Espírito Santo, Curso de Nutrição, Departamento de Educação Integrada em Saúde. Vitória, ES, Brasil.

2 Universidade Federal do Espírito Santo, Programa de Pós-graduaçãa em Nutriçãa e Saúde, Departamento de Educação Integrada em Saúde. Vitória, ES, Brasil.

Correspondência / Correspondence Jackline Freitas Brilhante de São José, Departamento de Educação Integrada em Saúde, Universidade Federal do Espírito Santo, Av. Marechal Campos, 1468, Maruípe, Vitória, ES, Brasil. CEP 29.040-090

E-mail: jackline.jose@ufes.br

\section{Resumo}

Objetivo: Avaliar o nível de conhecimento, atitudes e práticas de manipuladores de alimentos de serviços de alimentação. Materiais e Métodos: Trata-se de estudo transversal feito com manipuladores de alimentos de 15 serviços de alimentação, sendo que participaram da pesquisa 75 indivíduos. Foi aplicado um questionário contendo questões sociodemográficas e para avaliação do conhecimento, atitudes e práticas (CAP). O questionário autoaplicável continha 30 questões relacionadas às boas práticas na manipulação dos alimentos. Foi considerada adequada a nota igual ou superior a 70 \% para a avaliação de cada bloco e do questionário por completo. Os dados foram analisados com auxílio do software SPSS, versão 22. Resultados e Discussão: $64 \%$ dos manipuladores exerciam atividades em restaurantes comerciais e $84 \%$ eram do sexo feminino. Quanto ao nível de escolaridade, $52 \%$ dos manipuladores tinham o ensino médio completo. Quanto às questões sobre CAP, no bloco de conhecimento e atitudes foram observadas menores pontuações quando comparado ao bloco de práticas. A média de pontuação no questionário foi igual a $54,9 \pm 4,18$. Falhas quanto ao entedimento e conhecimento sobre cuidados com os alimentos podem diminuir o nível de consciência sobre manipulação adequada e culminar em uma falsa ideia de segurança. Conclusão: Os manipuladores apresentaram falhas na avaliação do conhecimento; ressalta-se, assim, a necessidade de treinamento, de modo a agregar informações para promover melhorias de desempenho nas atividades executadas.

Palavra-chave: Boas Práticas de Fabricação. Serviços de alimentação. Qualidade dos alimentos. Manipulação de alimentos. Controle de qualidade. 


\section{Abstract}

Aim: Assessing the level of knowledge, attitudes and practices of food handlers working in food services. Materials and Methods: Cross-sectional study conducted with food handlers from 15 food services: 75 individuals, in total. A questionnaire containing sociodemographic, as well as knowledge, attitudes and practices (KAP) evaluation questions was applied. The self-administered questionnaire comprised 30 questions about good food-handling practices. Scores equal to or higher than $70 \%$ were considered adequate to evaluate each block and the questionnaire as a whole. Data were analyzed in the SPSS software version 22. Results and Discussion: $64 \%$ of the herein evaluated food handlers performed commercial restaurant activities, $84 \%$ were women, and $52 \%$ had high school education. With respect to the KAP questions, the knowledge and attitude blocks recorded lower scores than the practice block. The mean score in the questionnaire was $54.9 \pm$ 4.18. Failures in the understanding and knowledge about food handling may lead to lower awareness about proper handling procedures and result in false ideas about food safety. Conclusion: Food handlers presented food handling-knowledge deficiencies; therefore, it is necessary training them so they can aggregate information to improve their performance.

Keywords: Good Manufacturing Practices. Food Service. Food quality. Food handling. Quality control.

\section{Introdução}

Nas últimas décadas, muitos países sofreram mudanças no status socioeconômico. Essas mudanças, em parte, levaram a um crescimento significativo na popularidade dos alimentos preparados fora de casa., ${ }^{1,2}$

A realização de refeições fora do lar teve crescimento considerável, tornando-se hábito de muitos, o que permitiu a ampliação do setor de serviços de alimentação, dentre os quais as Unidades de Alimentação e Nutrição (UAN). Estas possuem como objetivo oferecer refeições saudáveis para populações específicas, como funcionários de empresas, escolas, instituições filantrópicas, enquanto as Unidades Produtoras de Refeições (UPR) são restaurantes comerciais, padarias, lanchonetes, dentre outros. Diante desse crescimento e valorização do setor, houve aumento da competitividade e preocupação por parte dos consumidores quanto à qualidade sanitária e nutricional dos alimentos. Assim, é primordial que os estabelecimentos busquem melhorar a qualidade dos serviços oferecidos. ${ }^{3-8}$ 
O controle das condições higiênico-sanitárias nos locais em que os alimentos são manipulados constitui um ponto crítico, uma vez que contaminações de diferentes fontes podem ser introduzidas nas diversas etapas do preparo do alimento. A presença de micro-organismos patogênicos nos alimentos está associada à ocorrência de doenças de origem alimentar (DOA), que são umas das principais consequências da falta de controle higiênico-sanitário nesse setor, onde são encontrados perigos biológicos, físicos e químicos. ${ }^{9}$

Deste modo, o aumento na quantidade de refeições fornecidas gera maior preocupação com as estratégias para contribuir para a qualidade dos alimentos. O aprimoramento de ações de controle sanitário na área de alimentos, com o objetivo de controlar e minimizar os riscos originados pela ingestão de alimentos contaminados, torna-se essencial. ${ }^{10}$ De acordo com o Ministério da Saúde, os surtos notificados por DOA mostraram que, em 2010, 498 casos foram relatados e em 2011 foram notificados 795 casos, com acréscimo nesse período de 297 casos, o que se manteve até o ano de 2012, com 795 casos. $^{11}$

A manipulação inadequada é apontada como a principal causa de surtos envolvendo alimentos contaminados. Constantemente, surtos têm sido relatados em diversos tipos de serviços de alimentação, como em restaurantes, por exemplo. ${ }^{12-14}$ Neste contexto, o manipulador de alimentos tem importância crucial, pois durante as etapas do processo de produção de alimentos, ele pode facilitar e até provocar a disseminação de micro-organismos deterioradores e/ou patogênicos no ambiente de trabalho. ${ }^{15,16}$

Estudos conduzidos em diferentes estados do país mostram que os manipuladores de alimentos não estão completamente preparados, o que os relaciona diretamente com a contaminação dos alimentos, devido a doenças, maus hábitos de higiene e práticas inadequadas no processo produtivo de refeições. ${ }^{17}$ Durante a manipulação, o manipulador é considerado o principal responsável pela contaminação, sobretudo devido à falta de orientação e capacitação. É necessário melhorar a qualidade dos produtos e serviços, bem como capacitar os manipuladores de alimentos para que adquiram hábitos higiênico-sanitários adequados e os apliquem no dia a dia. ${ }^{12}$

A avaliação de conhecimentos, atitudes e práticas é importante para permitir melhor planejamento dos treinamentos dos manipuladores. ${ }^{18}$

Diante do exposto, o presente estudo teve como objetivo avaliar o nível de conhecimento, atitudes e práticas dos manipuladores de serviços em alimentação.

\section{Material e métodos}

Trata-se de estudo transversal, no qual a coleta de dados ocorreu entre julho e outubro de 2015, em restaurantes comerciais e em uma unidade de alimentação e nutrição localizados em Vitória- 
ES, com amostragem por conveniência. Os estabelecimentos foram contatados por meio de cartaconvite para apresentação dos objetivos da pesquisa; em seguida, solicitou-se a carta de anuência para participação da pesquisa. Os manipuladores de alimentos que concordaram em participar da pesquisa assinaram o Termo de Consentimento Livre e Esclarecido (TCLE). A pesquisa foi aprovada por um comitê de ética em pesquisa, registrado sob número 41393714.5.0000.5060.

\section{Avaliação do conhecimento, atitudes e práticas}

Para avaliar o conhecimento, atitudes e práticas (CAP), foi elaborado um questionário estruturado autoaplicável, considerando que todos os participantes da pesquisa eram alfabetizados, baseado em estudos com a mesma temática. ${ }^{19-22} \mathrm{O}$ conteúdo das questões baseou-se, ainda, na legislação atual sobre boas práticas na manipulação dos alimentos (RDC 216/2004). ${ }^{23}$

Para aplicação dos questionários, foram definidos os dias e horários conforme disponibilidade do local, sendo na maioria das ocasiões realizadas no período da tarde, após o término do expediente dos manipuladores. Os questionários foram distribuídos e preenchidos sem interferência de qualquer pessoa; em média, o tempo de aplicação foi de 20 minutos para cada participante.

A primeira parte do questionário teve como objetivo avaliar o conhecimento dos manipuladores sobre a segurança dos alimentos. Perguntas relacionadas às práticas diárias de manipulação de alimentos foram apresentadas, abordando higiene pessoal, higiene dos alimentos, contaminação cruzada, controle de temperatura, descongelamento de alimentos e higiene ambiental. Para a resposta, foi apresentada uma das três opões possíveis: "sim", "não" e "não sei”. A ordem de "sim" e "não" como resposta como correta foi aleatorizada e não seguiu um padrão. Um ponto foi atribuído para cada resposta correta, e zero para cada resposta incorreta ou "não sei". A faixa de escore de conhecimento situou-se entre 0 e $10 .{ }^{22}$

A segunda parte do questionário incluiu dez perguntas para avaliar as atitudes dos manipuladores de alimentos em relação à segurança dos alimentos, incluindo perguntas sobre a importância dos procedimentos de higiene, sua responsabilidade como manipuladores de alimentos para evitar doenças de origem alimentar, além da importância de aprender mais sobre a segurança alimentar. Os manipuladores de alimentos indicaram o nível de concordância de uma escala de três pontos: "concordo", "não sei" e "discordo". O intervalo de pontuação atitude foi entre 0 e $10 .{ }^{22}$

Na última parte do questionário, foram avaliadas as práticas autorrelatadas dos manipuladores. Foram incluídas dez perguntas sobre práticas diárias, abordando higiene pessoal, higiene dos alimentos, contaminação cruzada, controle de temperatura, descongelamento de alimentos e higiene ambiental. Nesta parte do questionário foi adotada uma escala de cinco pontos, que variou de $1=$ Nunca, $2=$ Raramente, $3=$ Às vezes, $4=$ Muitas vezes e $5=$ Sempre. As práticas foram pontuadas entre 10 e $50 .^{22}$ 
Considerou-se adequada a obtenção de nota igual ou superior a $70 \%$ para a avaliação de cada bloco e do questionário por completo.

\section{Análise de dados}

Os dados foram armazenados em planilhas no Microsoft Excel e, em seguida, analisados com auxílio do software SPSS, versão 22. Para avaliação da normalidade, utilizou-se Kolmogorov-Smirnov. Como os dados não apresentaram distribuição normal, a avaliação obtida para conhecimento, atitudes e práticas foi analisada utilizando-se o teste de correlação de Spearman, considerando p! 0,05. Foi realizada estatística descritiva com uso de média, desvio-padrão e valores percentuais para dados referentes a pontuação e características sociodemográficas. Foi conduzido também teste de associação qui-quadrado de Pearson $\left(\mathrm{X}^{2}\right)$ para avaliar possível relação entre características sociodemográficas e nível de conhecimentos, atitudes e práticas dos manipuladores de alimentos.

\section{Resultados e discussão}

Foram contatados 27 estabelecimentos, sendo 25 restaurantes e 2 UANs. Destes estabelecimentos, 14 restaurantes comerciais e uma UAN aceitaram participar da pesquisa. Dos 142 manipuladores convidados, 75 concordaram participar. As informações referentes às variáveis sociodemográficas dos manipuladores estão dispostas na tabela 1.

O maior número de participantes $(n=48)$ exercia atividades em restaurantes comerciais e a maioria $(84,0 \%)$ era do sexo feminino. Este foi um resultado semelhante ao estudo de Da Cunha et al. ${ }^{22}$ e Tan et al., ${ }^{24}$ nos quais também houve maior representação do sexo feminino entre os participantes. De acordo com Ferreira et al., ${ }^{25}$ as atividades relacionadas a cuidados dos alimentos e da alimentação são caracterizadas como trabalho feminino e, por consequência, a mulher ocupa esse espaço no mercado de trabalho. Além disso, as mulheres são mais facilmente empregadas nesse tipo de função, devido à similaridade com as tarefas domésticas, como o ato de cozinhar. ${ }^{22}$

O nível de escolaridade mais representativo foi até o ensino médio completo (52\%), como também foi observado no estudo de Devides et al., ${ }^{26}$ onde $55 \%$ manipuladores informaram possuir o $2^{2}$ grau completo. Há relação direta entre o nível educacional de manipuladores de alimentos e a adoção de boas práticas. Deste modo, o acesso a essas informações torna-se essencial para o planejamento de cursos de capacitação. ${ }^{26,27}$

Quando questionados sobre a participação em treinamentos 26,7\% alegaram nunca terem participado. Resultado semelhante foi observado por Da Cunha et al., ${ }^{22}$ onde 31,7\% dos manipuladores participantes não receberam qualquer tipo de treinamento. Diante disto, vale 
ressaltar que a capacitação dos manipuladores de alimentos pode ser entendida como uma estratégia de aprendizagem previamente planejada que visa aumentar o conhecimento sobre as atividades desempenhadas, bem como mudanças permanentes de práticas e atitudes. ${ }^{28}$

Tabela 1. Características sociodemográficas dos manipuladores de alimentos de restaurantes comerciais e UAN de Vitória-ES, 2015.

\begin{tabular}{ccc}
\hline VARIÁVEL & $\mathrm{N}$ & $\%$ \\
\hline Sexo & 63 & 84,0 \\
Feminino & 12 & 16,0 \\
Masculino & & \\
Idade (anos) & 50 & 66,7 \\
Até 39 & 13 & 17,3 \\
$40-49$ & 12 & 16,0 \\
$>$ 50 & & \\
Escolaridade & 32 & 42,7 \\
Até ensino fundamental completo & 39 & 52,0 \\
Até ensino médio completo & 4 & 5,3 \\
Até ensino superior completo & & \\
Experiência anterior na área & 68 & 90,7 \\
Sim & 7 & 9,3 \\
Não & & \\
Treinamento & 20 & 26,7 \\
Nunca & 25 & 40,3 \\
Uma vez & 30 & 64,0 \\
Mais de uma vez & & 36,0 \\
Local de trabalho & 48 & \\
Restaurante comercial & 27 & \\
UAN & &
\end{tabular}

O treinamento é uma exigência legal no ambiente de produção de alimentos..$^{23}$ Além disso, é importante que a capacitação dos manipuladores de alimentos seja contínua, pois, embora no setor de alimentação seja comum a contratação de profissionais sem experiência na função $0^{20,29}$, no presente estudo a experiência na função foi relatada por $68 \%$ dos participantes.

Na tabela 2, consta a média da pontuação obtida em cada bloco de perguntas e no questionário por completo. Observou-se que, na avaliação de conhecimentos, os manipuladores obtiveram menor pontuação. Este resultado pode gerar questionamento acerca das atitudes relatadas 
pelos entrevistados, uma vez que se para este bloco houve maior pontuação. Os manipuladores afirmaram ter atitudes que auxiliam na produção de alimentos seguros, porém responderam incorretamente questões diretamente relacionadas ao controle de qualidade de alimentos. Da Cunha et al. ${ }^{22}$ observaram notas inferiores para conhecimento $(5,4 \pm 1,8)$ e prática $(36,0 \pm 3,6)$; entretanto, para atitude as notas obtidas foram superiores $(9,0 \pm 1,1)$.

Tabela 2. Pontuação obtida na avaliação do conhecimento, atitudes e práticas dos manipuladores de alimentos em serviços de alimentação de Vitória-ES, 2015.

\begin{tabular}{ccc}
\hline Variável & Média \pm Desvio Padrão & $\begin{array}{c}\text { Intervalo } \\
\text { Mínimo e Máximo }\end{array}$ \\
\hline Conhecimento & $6,7 \pm 1,45$ & $4-10$ \\
Atitude & $8,1 \pm 1,23$ & $4-10$ \\
Prática & $40,6 \pm 3,2$ & $34-48$ \\
Questionário & $54,9 \pm 4,18$ & $47-64$ \\
\hline
\end{tabular}

A baixa correlação entre as pontuações de conhecimento e de atitudes (tabela 3) indica que o nível de conhecimento sobre segurança dos alimentos pelos manipuladores pode influenciar as atitudes de manipulação de alimentos, ou seja, um baixo nível de conhecimento culmina em atitudes inadequadas. A atitude é a tendência psicológica manifestada em nível de concordância ou desacordo sobre um certo fato/tema. A prática é a execução de uma atividade ou método particular de forma habitual ou regular. ${ }^{28}$ Segundo Clayton et al.,${ }^{29}$ funcionários de serviços de alimentação com bom conhecimento sobre segurança dos alimentos nem sempre têm atitudes adequadas e ou aplicam esse conhecimento na prática.

Tabela 3. Correlação entre as pontuações obtidas em conhecimento, atitudes e práticas de manipuladores de alimentos em serviços de alimentação de Vitória-ES, 2015

\begin{tabular}{ccccccc}
\hline & Conhecimento & $p$ & Atitude & $p$ & Prática & $p$ \\
\hline Conhecimento & & & & & & \\
Atitude & $\mathbf{0 , 2 8 0 *}$ & 0,015 & & & \\
Prática & 0,178 & 0,127 & 0,053 & 0,653 & \\
\hline
\end{tabular}

*A correlação é significativa no nível 0,05 . 
Com relação à frequência dos manipuladores que obtiveram a pontuação considerada adequada (figura 1), apenas 32 profissionais atingiram a nota adequada na avaliação geral do questionário. É possível observar que, quando comparado aos demais blocos de avaliação, o bloco sobre conhecimento em segurança de alimentos apresentou o menor índice de obtenção da nota adequada (n=38). O bloco para avaliação de práticas foi o que apresentou melhor desempenho pelos manipuladores $(\mathrm{n}=74)$. Segundo Soares et al., ${ }^{30}$ as práticas autorrelatadas tendem a ser aumentadas pelo indivíduo que responde ao questionário, ou seja, o manipulador respondeu o que era esperado, e não de fato o que ele faz no dia a dia no ambiente de produção de refeições.

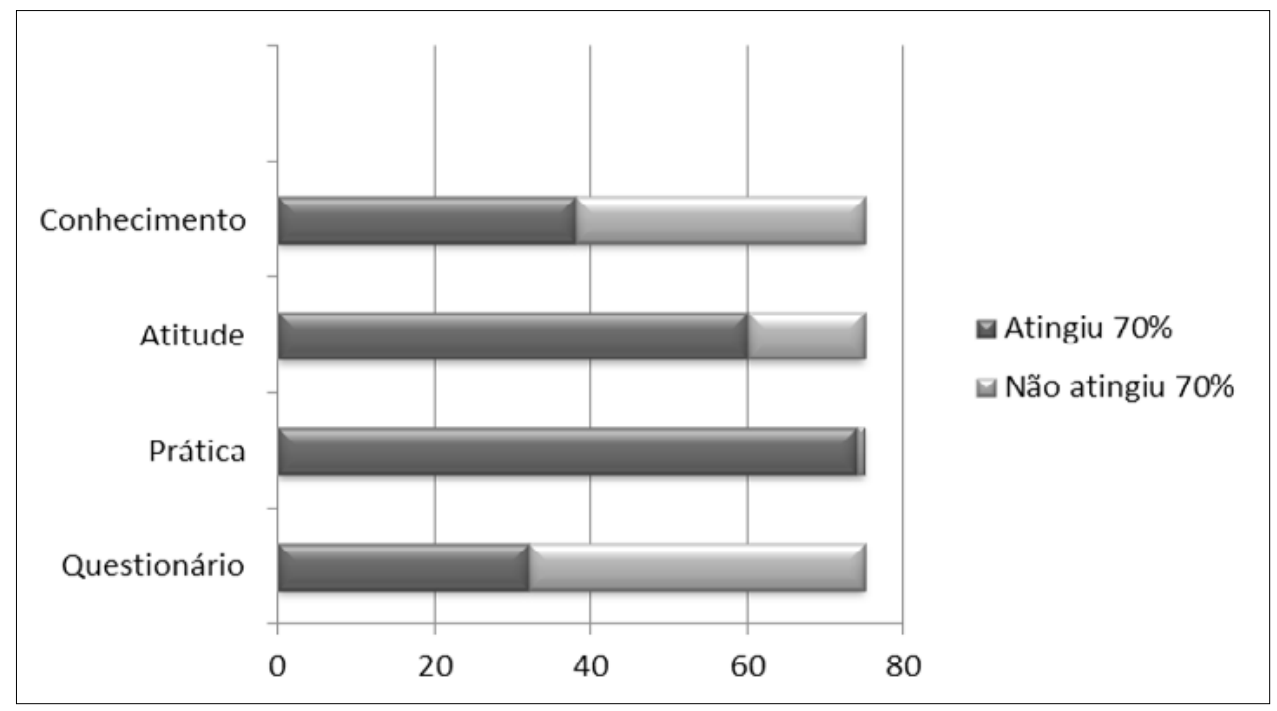

Figura 1. Distribuição dos manipuladores de alimentos segundo a pontuação adequada obtida na avaliação do questionário. Vitória, 2015.

As questões com maior percentual de erro foram sobre higiene das mãos (93,3\%), transmissão de doenças pela água (64\%) e descongelamento de alimentos (56\%). Da Cunha et al. ${ }^{22}$ também observaram elevado percentual de erro para a pergunta sobre higienização de mãos (81 \%). Segundo a RDC $216,{ }^{23}$ os lavatórios devem possuir sabonete líquido inodoro antisséptico ou sabonete líquido inodoro e produto antisséptico, para permitir a adequada higienização de mãos.

A questão sobre o risco no reaquecimento dos alimentos apresentou elevado percentual de acerto (77,3\%). Resultado semelhante ao observado por Soares et al. ${ }^{31}$ que verificaram que manipuladores têm consciência do risco ao reaquecer os alimentos $(93,4 \%)$. 
As questões com maior percentual de acertos foram aquelas sobre o público de risco para intoxicação alimentar (84\%) e os riscos para contaminação de alimentos decorrentes da presença de doenças como diarreia, gripe e dor de garganta no manipulador de alimentos (89,3\%). Falhas quanto ao entedimento e conhecimento sobre cuidados com os alimentos podem diminuir o nível de consciência sobre manipulação adequada e culminar em uma falsa ideia de segurança. ${ }^{21}$

Além do conhecimento, a atitude é também fator crucial que pode influenciar nos comportamentos e práticas relacionados à segurança dos alimentos. ${ }^{21}$ Do total de questões referentes ao bloco sobre atitudes, em $70 \%(\mathrm{n}=7)$ das questões apresentadas foram observados percentuais de respostas positivas superiores a $90 \%$. Tal resultado se assemelha ao observado por outros autores, ${ }^{19,22}$ os quais observaram percentuais superiores a $90 \%$ em nove das dez questões.

As questões com maior percentual de respostas positivas no bloco de atitudes foram sobre higiene das mãos após uso de sanitários e a manipulação de lixo $(98,7 \%)$, verificação da validade e integridade de produtos $(98,7 \%)$ e importância do aprendizado sobre manipulação segura dos alimentos $(97,3 \%)$.

Com relação à questão sobre higienização de mãos, resultado diferente foi observado por Bas et al. ${ }^{32}$ que identificaram que apenas $21,2 \%$ dos manipuladores de alimentos relataram a necessidade de lavar as mãos após ir ao banheiro, manusear alimentos crus e antes de manusear alimentos prontos. A higienização correta das mãos é importante, pois sabe-se que os manipuladores podem ser fontes de micro-organismos patogênicos, devido a falhas na higiene pessoal e contaminação cruzada. $^{32}$

A questão sobre o risco de intoxicação alimentar decorrente do preparo de alimentos com antecedência e armazenamento inadequado obteve elevado percentual de respostas negativas (45,3\%). Segundo a RDC 216/2004, o armazenamento e o transporte do alimento preparado, da distribuição até a entrega ao consumo, devem ocorrer em condições de tempo e temperatura que não prejudiquem a qualidade higienicossanitária. ${ }^{23}$

Outra questão com percentual elevado de respostas negativas foi sobre a presença de ferimentos, contusões ou lesão nas mãos e manipulação de alimentos (29,3\%). No estudo de Ferreira et al., ${ }^{25}$ $95,4 \%$ e $88,6 \%$ dos manipuladores responderam positivamente a questões sobre a importância da saúde do trabalhador e o afastamento das atividades laborais por motivo de doença, e sobre a exigência de realização de exames periódicos para avaliação do estado de saúde, respectivamente. Ainda segundo a RDC 216/2004, ${ }^{23}$ o manipulador de alimentos deve ser afastado do trabalho, caso esteja doente, destacando-se que exames admissionais e periódicos são indispensáveis para a contratação. O acompanhamento do estado de saúde do trabalhador é de fundamental importância na prevenção de contaminação do alimento. ${ }^{23,25}$ 
As práticas positivas mais frequentes foram sobre a higiene pessoal (98,7\%), higiene com os uniformes $(93,3 \%)$ e o cuidado ao manter o cabelo completamente coberto durante o trabalho $(90,7 \%)$. Veiga et al. ${ }^{33}$ afirmaram que a higiene pessoal influencia a higiene dos alimentos e pode afetar a qualidade e a segurança dos mesmos.

Entre as práticas negativas mais evidenciadas, 50,7\% dos participantes relataram descongelar alimentos em temperatura ambiente. $\mathrm{O}$ descongelamento inadequado favorece a multiplicação microbiana nos alimentos e, consequentemente, contribui para a ocorrência de toxinfecções alimentares. Resultado semelhante foi encontrado no estudo de Da Cunha et al., ${ }^{22}$ sendo a mais inadequada prática citada pelos manipuladores, na qual 34,6\% dos entrevistados relataram sempre executar o descongelamento desta forma.

Além disso, 14,7\% dos manipuladores afirmaram ir ao trabalho quando estão com diarreia, outra doença ou apresentam cortes e feridas nas mãos. Este resultado pode indicar que os manipuladores desconhecem os riscos de manipular os alimentos em situações de enfermidades, e também receio quanto ao afastamento do trabalho e possíveis prejuízos salariais.

\section{Conclusão}

A falta do conhecimento em boas práticas de manipulação gera deficiência nas atitudes, pois informações básicas como higiene das mãos, transmissão de doenças pela água e descongelamento de alimentos obtiveram os maiores percentuais de erro no presente estudo. As práticas tiveram percentual de acerto alto e considerado como satisfatório. Neste caso, cabe ressaltar que o fato de o manipulador no início da pesquisa concordar em participar e de o questionário ser autoaplicável podem ter influenciado o alto número de respostas consideradas corretas. Ou seja, o manipulador respondeu o que era esperado, e não de fato o que ele faz no dia a dia. Assim, pode-se considerar que a avaliação de CAP, por estar limitada a respostas dadas pelos manipuladores, pode ser apenas um primeiro passo para entendimento do comportamento dos mesmos.

Faz-se necessária a realização de estudos mais aprofundados, considerando a possível influência de fatores psicossociais e da experiência no comportamento dos manipuladores na rotina de produção dos alimentos.

\section{Agradecimentos}

Os autores agradecem a todos os estabelecimentos e manipuladores de alimentos participantes desta pesquisa. 


\section{Colaboradores}

Pagotto HZ e LG Espíndula participaram da concepção, coleta dos dados e interpretação dos dados e redação do artigo; Vitória AG e Machado MCMM participaram da interpretação dos dados, da redação do artigo e da sua versão final; São José JFB trabalhou em todas etapas deste a concepção do estudo, orientação até a revisão da versão final do artigo.

Conflito de interesses: Os autores declaram não haver conflito de interesse.

\section{Referências}

1. Chukuezi CO. Food safety and hygienic practices of street food vendors in Owerri, Nigeria. Studies in Sociology of Science. 2010; 1(1):50-57.

2. Omemu A, Aderoju ST. Food safety knowledge and practices of street food vendors in the city of Abeokuta, Nigeria. Food Control. 2008; 19(4):396-402.

3. Bachelli MLB, Villa F, Oliveira IBN, Rodrigues KRM, Salay E. Iniciativas de implantação de selos de qualidade em restaurantes no Brasil. Hig. Alim. 2004; 18(121):20-25.

4. Sneed J, Strohnehn CH. Trends impacting food safety in retail foodservice: Implications for dietetics practice. J Am Diet Assoc. 2008; 108(7):1170-1177.

5. Alves MG, Ueno M. Restaurantes self-service: segurança e qualidade sanitária dos alimentos servidos. Rev Nutr. 2010; 23(4):573-580.

6. Abreu E, Spinelli, MGN, Pinto, AMS. Gestão de Unidades de Alimentação e Nutrição:um modo de fazer. São Paulo: Metha; 2011.

7. Ungku FU, Boo HC, Sambasivan M, Salleh R. Foodservice hygiene factors - the consumer perspective. Int J Hosp Manag. 2011; 30(1):38-45.

8. Gormley FJ, Rawal N, Little CL. Choose your menu wisely: cuisine-associated food-poisoning risks in restaurants in England and Wales. Epidemiol Infect. 2011; 140(6):1-11.

9. São José JFB. Contaminação microbiológica em serviços de alimentação. Nutrire: Rev Soc Bras Alim Nut. 2012; 37:78-92.

10. São José JFB, Coelho AIM, Ferreira KR. Avaliação das boas práticas em unidade de alimentação e nutrição no município de Contagem-MG. Alim Nutr. 2011; 22(3):479-487.

11. Brasil. Ministério da Saúde. Secretaria de Vigilância em Saúde. Departamento de Análise de Situação de Saúde. Plano de ações estratégicas para o enfrentamento das doenças crônicas não transmissíveis (DCNT) no Brasil 2011-2022. Brasília: Ministério da Saúde; 2011.

12. Chan SF, Chan ZCY. A review of foodborne disease outbreaks from 1996 to 2005 in Hong Kong and its implications on food safety promotion. J Food Safety. 2008; 28:276-299.

13. Costalunga S, Tondo EC. Salmonellosis in Rio Grande do Sul, Brazil, 1997 to 1999. Braz J Microbiol. 2002; 33(4):342-346. 
14. Xue JH, Zhang WJ. Understanding China's food safety problem: an analysis of 2387 incident of acute foodborne illness. Food Control 2013; 30(1):311-317.

15. Green LR, Selman CA, Radke V, Ripley D, Mack JC, Reimann DW, Stigger T, Motsinger M, Bushnell L. Food worker hand washing practices: an observation study. J Food Protection. 2006; 69(10):2417-2423.

16. Egan MB, Raats MM, Grubb SM, Eves A, Lumbers ML, Dean MS, et al. A review of food safety and food hygiene training studies in the commercial sector. Food Control 2007; 18:1180-1190.

17. Martins SCS, Martins CMM, Alburquerque LMB, Faheina Jr G. Perfil da resistência de cepas de Staphylococcus coagulase positiva isoladas de manipuladores de alimentos. Boletim CEPPA. 2009; 27(1):43-52.

18. Ricke S, Donalds J, Phillips C. The role of training strategies in food safety performance: knowledge, behavior and management. In: Ricke C, Donaldson JR, Phillips CA. Food Safety emerging issues, technologies and systems. London: Elsevier; 2015.

19. Ansari-Lari M, Soodbakhsh S, Lakzadeh L. Knowledge, attitudes and practices of workers on food hygienic practices in meat processing plants in Fars, Iran. Food Control. 2009; 21(3):260-263.

20. Mello AG, Gama MP, Marin VA, Colares LGT. Conhecimento dos manipuladores de alimentos sobre boas práticas nos restaurantes públicos populares do Estado do Rio de Janeiro. Braz J Food Technol. 2010; 13(1):60-68.

21. Sani NA, Sion ON. Knowledge, attitudes and practices of food handlers on food safety in food service operations at the Universiti Kebangsaan, Malaysia. Food Control. 2014; 37(1):210-217.

22. Cunha DT, Stedefeldt E, Rosso VV. The role of theoretical food safety training on Brazilian food handlers' knowledge, attitude and practice. Food Control. 2014; 43:167-174.

23. Brasil. Ministério da Saúde. Secretaria de Vigilância Sanitária. Resolução RDC no 216, de 15 de setembro de 2004. Regulamento Técnico de Boas Práticas para Serviços de Alimentação. Diário Oficial da União. 16 set. 2004.

24. Tan SL, Bakar FA, Karim MSA, Lee HY, Mahyudin NA. Hand hygiene knowledge, attitudes and practices among food handlers at primary schools in Hulu Langat district, Selangor (Malaysia). Food Control. 2013; 34:428-435.

25. Ferreira JS, Cerqueira ES, Carvalho JS, Oliveira, LC, Costa WLR, Almeida RCC. Conhecimento, atitudes e práticas em segurança alimentar de manipuladores de alimentos em hospitais públicos de Salvador, Bahia. Rev Baiana Saúde Pública. 2013; 37(1):35-55.

26. Devides GGG, Maffei DF, Cantonazi MPLM. Perfil socioeconômico e profissional de manipuladores de alimentos e o impacto positivo de um curso de capacitação em Boas Práticas de Fabricação. Braz J Food Tecnh. 2014; 17(2):166-176.

27. Çakiroglu FP, Uçar A. Employees' Perception of Hygiene in the Catering Industry in Ankara (Turkey). Food Control. 2008; 19(1):09-15.

28. Ricke SC, Donaldson JR, Phillips CA, editors. Food safety: emerging issues, technologies and systems. London: Academic Press; 2015. 
29. Clayton DA, Griffith CJ, Price P, AC. Peters AC. Food handlers beliefs and self: reported pratices. Int J Environm Health Res. 2002; 12(1): 25-39.

30. Mcintyre L, Vallaster L, Wilcott L, Henderson SB, Kosatsky T. Evaluation of food safety knowledge, attitudes and self-reported hand washing pratices in foodsafe trained and untrained food handlers in British Columbia, Canada. Food Control. 2013; 30(1):150-156.

31. Soares LS, Almeida RCC, Cerqueira ES, Carvalho JS, Nunes IL. Knowledge, attitudes and practices in food safety and the presence of coagulase positive staphylococci on hands of food handlers in the schools of Camaçari, Brazil. Food Control. 2012; 27(1):206-213.

32. Bas M, Ersun AS, Kivanc G. The evaluation of food hygiene knowledge, attitudes, and practices of food handlers in food businesses in Turkey. Food Control. 2006; 17(4):317-322.

33. Veiga CF, Doro DL, Oliveira KMP, Bombo DL. Estudo das condições sanitárias dos estabelecimentos comerciais de manipulação de alimentos do município de Maringá, PR. Hig Alim. 2006; 20(138):28-36.

Recebido: 13 de outubro, 2017

Revisado: 07 de março, 2018

Aceito: 22 de março, 2018 
